\title{
Self-management of vaginal pessaries for pelvic organ prolapse
}

Rohna Kearney, Claire Brown

Cambridge University Hospitals NHS Trust

\begin{abstract}
Two thirds of women opt to use a vaginal pessary initially to manage the symptoms of pelvic organ prolapse. In the UK most women attend a health care professional at least every six months to change the pessary. This represents a significant burden both economically to the health care system and personally for the woman. Annually there are more than 300 appointments for pessary changes at our hospital. We developed a programme to teach women to self-manage their pessaries with the aim of improving patient experience and reducing outpatient attendances to free up outpatient capacity for new referrals. A physiotherapist was recuited to deliver this programme involving a one to one training session supplemented with written materials and an online video. Women using pessaries were offered the option of selfmanagement.
\end{abstract}

Eighty-eight women aged between 29 to 84 years enrolled in the programme. Sixty-three women (73\% of those enrolled) successfully continued with self-management at six months, creating 126 extra outpatient appointment capacity in one year alone. Women self-managing reported higher levels of convenience ( $94 \%$ vs $81 \%$ ), accessibility ( $97 \%$ vs $73 \%$ ), support ( $100 \%$ vs. $83 \%$ ), and comfort ( $86 \%$ vs. $53 \%$ ) than those attending the hospital for GP practice for pessary change.

Self-management appears to be an acceptable option for many women using vaginal pessaries, with personal benefits to the women and economic benefits to the hospital and commissioners.

\section{Problem}

While there is evidence that vaginal pessaries are a valid treatment option for pelvic organ prolapse there is very little data regarding the optimal management pathway for women using pessaries. Women in North America are routinely taught how to change their own pessary. A survey by the American Urogynaecological Society in 2000 reported $57 \%$ of its members taught women selfmanagement.[1] However, practice in the UK is very different; a recent multi-professional survey reported that only $17 \%$ of health care professionals offer the option of pessary self-management[2] This means that most women in the UK attend a healthcare professional for a pessary change every six months. This represents a significant use of healthcare resources. Women are not being offered the option of self-managing their condition and are obliged to attend appointments for pessary changes. This is one of the factors that may contribute to women deciding to opt for surgical management.

\section{Background}

Pelvic organ prolapse (POP) is a common condition with a lifetime risk of surgery in the general female population of up to $19 \%$.[3] Vaginal pessaries are an effective way to manage prolapse symptoms and may be offered to women with symptomatic prolapse as an alternative to surgery. There is no data available for pessary changes in primary care, although Dr Foster data records over 46,000 pessary changes in hospitals in England during 2013. This clearly represents a significant use of healthcare resources as well as the personal and economic costs to women having to attend for an appointment. Reasons for declining or discontinuing pessary management include experience of discomfort at pessary changes, inconvenience of attending appointments, discharge, bleeding, and reduced sexual activity[4,5] These problems can be resolved with self-management as the woman is able to remove, clean, and insert the pessary at her convenience. At our institution over 300 pessary changes are performed each year.

\section{Baseline measurement}

Outpatient utilisation of 307 appointments annually for pessary changes. Self-management not offered or taught in our institution or in primary care.

\section{Design}

A focus group of pessary users was formed to help design the selfmanagement programme. This group allowed us to learn from patient experiences with pessary changes and met to discuss the self-management learning process, timing of follow-up phone calls, review of written information leaflets, and the self-management pathway. Stakeholders were contacted and invited to discuss the programme. A women's health physiotherapist was recruited and trained in pessary use by the gynaecologist. The teaching programme was then developed by the gynaecologist, physiotherapist, and hospital manager following extensive consultation with pre-existing pessary users via focus groups and stakeholders. Self-management was initially offered by written letter to women using a ring or sieve pessary at our institution over the past year. Women attending the gynaecology clinic who chose 
pesssary use for management of their prolapse symptoms were offered self-management from the beginning of the programme. Referrals were also accepted from general practitioners, physiotherapists, and nurse continence advisors. Women could self refer if they had a pessary already in situ.

\section{Strategy}

The service was advertised by newsletters, hospital web pages, communication emails, and through professional interest groups. A questionnaire was used to assess patient satisfaction of pessary management using a Likert scale. The questionnaire was given to women who declined self-management and remained in the doctor management group (DM) and to women who had been in the selfmanagement group (SM) for three months. Reasons for declining self-management were also recorded.

The teaching package was delivered by a specialist women's health physiotherapist. During a single 45-minute appointment, informed consent was obtained and the woman was shown and supervised in pessary change. Baseline levels of confidence in changing pessaries using a visual analogue scale (VAS) was recorded as well as ascertaining whether the women was considering surgical intervention for prolapse. This face to face training was supplemented with written information and an online teaching video. Feedback from the focus group on the information leaflets was used to inform the structure of the teaching video, with increased emphasis placed on simple diagrammatic presentation of prolapse and pessary in situ. Women received telephone follow-up at two weeks, one month, three months, and six months, which included repeat VAS reports of confidence (the patient was shown the VAS at initial appointment). At three months, the frequency of pessary changes and reasons for change were recorded. Women were also asked if they wished to continue with self-management, continue with a pessary but not self-management, discontinue pessary use altogether, or opt for surgery. Women who had considered surgery were asked again at six months. If women encountered complications such as bleeding or excessive vaginal discharge then they were rebooked to see a gynaecologist. A new pessary was posted to the patient once the current pessary was 12 months old.

During the study, the responses to telephone follow-up were studied and the frequency of telephone follow-up was reduced as patients reported that they felt adequately supported with one followup call.

\section{Results}

Eighty-eight women aged between 29 to 84 years accepted the offer of learning to self-manage (SM group). Sixty-four were recruited directly from clinic, 14 women were referred by their GP, four women from physiotherapists, two women self-referred. No women were recruited from nurse continence advisors. Only four out of 71 who were were invited by letter after review of clinic attendances the previous year responded to the invitation (response rate 6\%). Forty-six women remained in the doctor management group (DM group) and were aged between 40 to 92 years of age. The median duration of pessary use prior to being offered self-management in the DM group was 25 months compared with one month in the SM group.

Sixty-three women (73\%) were successfully continuing with selfmanagement at six months telephone follow-up. There was no difference between groups in the size of pessary but more women were self-managing a ring pessary rather than a sieve pessary (table 1).

Women in the SM group reported higher levels of comfort with changes ( $86 \%$ vs $53 \%$ ), convenience ( $94 \%$ vs $81 \%$ ), ability to access help ( $97 \%$ vs $73 \%$ ), and feeling supported (100\% vs $83 \%)$ than those in DM group. Ninety-seven percent of the SM group plan to continue pessary use in the long term for managing their prolapse symptoms compared with $70 \%$ in the DM group. Following six months of self-management, 17 out of 25 women previously considering surgery were no longer doing so. Women's confidence levels of changing their pessary remained constant during the time period following the appointment with the physiotherapist and at six months follow-up (figure 1). The frequency of pessary removal and insertion varied from twice a week to once every six months. Reasons for removal and insertion are listed in table 2.

One patient experienced an increase in vaginal discharge and was advised to remove the pessary less frequently. No other complications occurred in the SM group.

Women who did not respond to the invitation letter were contacted by telephone, 46 women gave reasons for declining the service (table 3).

Twenty-four women (27\%) stopped SM by month six: nine reported that poor manual dexterity precluded pessary insertion, one experienced bleeding and switched to doctor led care, one switched to a shelf pessary requiring doctor led care, 11 stopped using a pessary, and two women opted for surgery.

The key measures that we have used in understanding the cost of providing a doctor led pessary management service are staffing costs in clinic, the cost of replacement pessaries, and the tariff cost that commissioners pay for an outpatient follow up appointment.

This provided us with two separate costs: one to the commissioner ( 168 per patient per year) and one for the hospital trust ( $£ 140$ per patient per year). These costs can be calculated as savings for each individual patient that goes over to self management from doctor led care.

The cost of providing the self management service can be related directly to the cost of the physiotherapist or nurses time in teaching the patient. We have calculated that each patient will take approximately 1.5 hours to teach and then follow up, which equates to a one off cost of approximately $£ 30$ per patient. This is significantly lower than the cost per year to the Trust of doctor led care. We have summarised the cost impact of this below, based on a cohort of 50 patients moving to self management: 
Doctor led care $=£ 7,000$ cost to Trust and $£ 8,400$ cost to commissioners

Self management $=£ 1,500$ cost to Trust and no cost to commissioners

Self-management savings $=£ 5,500$ for Trust and $£ 8,400$ for commissioners.

The opportunity to scale these savings up across the region is significant as Dr Foster suggests that there were over 6,688 hospital clinic attendances across the East of England last year for pessary changes. Based on local practice this would suggest approximately 4,000 individual patients using pessaries in the region, which doesn't take into account the majority of pessary patients which are managed via GP practices. You can see that the opportunity to release savings if self management were to become embedded within the region is significant. Even if you were to take a very conservative estimate and only base savings on the direct tariff cost to the commissioners of those 6,688 attendances in clinic, then the opportunity is in excess of $£ 500,000$.

See supplementary file: ds3864.docx - "Figure 1 and Tables"

\section{Lessons and limitations}

The primary outcome of this project was an assessment of patient satisfaction with self-management of vaginal pessary for prolapse. We found that self-management was acceptable to two-thirds of our pessary user group when they were offered the option of self management at the beginning of pessary use. Those self-managing successfully reported higher levels of comfort with pessary use and were more likely to continue with pessary management in the long term compared with those who continued to attend a health care professional for pessary changes. However, we found that when women were offered the option of self-management after a longer period of pessary use they were less inclined to consider this. It may be that this group, having become accustomed to seeing a health professional for pessary changes, perceived that this was required to safely manage their condition. We did not market to stake-holders until six months into the project because we had expected more of our existing patients to want to try SM. Once we did, we noticed an increase in referrals.

At stakeholder engagement, concerns were expressed that "older" women would not be able to self-manage, however we did not find that this was a barrier and the age range in both SM and DM groups was similar. Many women in the SM group reported that they only use their pessaries intermittently, for example if going on a long walk, and value that self-management allows them to personalise and take control of the management of their prolapse symptoms. Patients have reported that they feel more empowered and in control through self-management.

The physiotherapist only worked two days a week which limited the speed at which we could raise the profile of the project internally. The physical environment of where our clinic was held meant integrating with other members was difficult because there were a limited number of clinic rooms being available. This sometimes meant that while we aimed to offer a "one stop" service where women could be taught SM straight after finding out about it in clinic, there wasn't always a room available for us to offer this service and so they would have to come back. A further barrier of the project was the reorganisation of our local primary care setting. This has delayed some of the discussions that we wanted to have earlier on in the project with commissioners about sustainability of the project.

Recruiting a physiotherapist to this project was not something that we had originally considered as we had initially seen this as a specialist nursing role. However, we believe that by having a physiotherapist (a role which traditionally focuses on rehabilitation and not medical intervention) at the heart of the service, we have been able to develop something which is truly focussed on empowering the patient to manage their own condition away from a healthcare setting. As this project demonstrated an improvement in patient experience and cost savings, the trust have committed to sustaining self-management support with a permanent physiotherapy role.

\section{Conclusion}

We have demonstrated that self-managing vaginal pessaries is acceptable to many women and represents an improvement in experience in managing their prolapse symptoms compared with doctor management. In addition, self-management is cost effective both for the hospital trust delivering the service and the commissioners.

\section{References}

1. Cundiff GW, Weidner AC, Visco AG, Bump RC, Addison WA. A survey of pessary use by members of the American Urogynaecological Society. Obstet Gynaecol 2000;95:931-35.

2. Bugge $C$, Hagen S, Thakar R. Vaginal pessaries for pelvic organ prolapse and urinary incontinence: a mulitprofessional survey of practice. Int Urogynaecol J 2013; 24:1017-24.

3. Smith FJ, Holman CD, Moorin RE, Tsokos N. Lifetime risk of undergoing surgery for pelvic organ prolapse. Obstet Gynaecol 2010;116:1096-100.

4. Sarma S, Ying T, Moore KH. Long-term vaginal ring pessary use: discontinuation rates and adverse event. BJOG 2009;116:1715-21.

5. Sulak $P$, Kuehl $T$, Shull B. Vaginal pessaries and their use in pelvic relaxation. J Rep Med 1993;38:919-23.

\section{Declaration of interests}

Rohna Kearney has accepted sponsorship to attend academic meetings from Astellas, Boston Scientific, and Pfizer. She has been paid speaker fees by Astellas.

Claire Brown has no conflict to declare. 
BMJ Quality Improvement Reports

\section{Acknowledgements}

Myfawny Champness has been the project manager and was involved in study design and grant submission.

Funding received from Health Foundation SHINE programme 2012. 